\title{
Assessing Access to Healthcare in Bulgaria
}

\author{
Assist. Prof. PhD Mincho Minev \\ Medical University - Varna, Varna, Bulgaria \\ mincho.minev@mu-varna.bg
}

\begin{abstract}
Assessing access to healthcare in a given country remains a challenging endeavor. Despite the frequent use of access as a dimension of the health system performance assessment frameworks, there is no agreed-upon set of indicators to evaluate it. This study aims to assess the access to healthcare in Bulgaria. The assessment is based on a set of the most commonly used indicators with publicly available data. Results show that timeliness and physical availability are not substantial barriers for obtaining health services in Bulgaria, as the values for most of the considered indicators are better than the EU average. The most significant barrier to access to healthcare in Bulgaria is the financial affordability. Data shows insufficient financial protection alongside other factors resulting in higher than the EU average level of unmet needs due to financial reasons. Policy measures focused on decreasing the share of private health expenditures ought to be exerted to reduce inequalities in access to healthcare services.
\end{abstract}

Keywords: access; Bulgaria; access indicators; performance assessment

JEL Code: I110; doi:10.36997/IJUSV-ESS/2019.8.1.141

\section{Въведение}

Едно от предизвикателствата пред съвременните здравни системи е осигуряването на адекватен достъп до здравни услуги. Справянето с това предизвикателство изисква предприемане на мерки, ограничаващи негативното влияние на различните бариери пред него. Отправна точка за разработването и прилагането на подобни мерки трябва да бъде комплексната и научнообоснована оценка на достьпа.

Въпреки че достъпът до здравни услуги е едно от най-често срещаните измерения при извършване на цялостна оценка на функционирането на здравната система (Braithwaite et al., 2017), липсва единна и общовалидна рамка за неговото измерване (Commission of the European Communities, 2009). В научната литература не съществува консенсус относно дефинирането на самото понятие за достъп до здравни услуги. Редица автори, изследвали въпроса, дават свои дефиниции, в които акцентират на една или друга негова характеристика (Aday and Andersen, 1974; Penchansky and Thomas, 1981; Murray and Frenk, 2000). Като обобщение на различните интерпретации може да се посочи, че достъпът се определя като връзката между нуждите от здравни услуги на населението, предлагането на тези услуги в здравната система и равнището на незадоволените потребности.

Достьпът до здравни услуги е многопластова категория. Адекватното му измерване и интерпретиране налага обхващане на отделните му елементи. В научната литература се срещат разнородни мнения относно техния брой и състав (Penchansky and Thomas, 1981; Clark, 1983; Evans, Hsu and Boerma, 2013; Saurman, 2016). Липсата на общоприето виждане по въпроса за елементите на достьпа до здравни услуги предполага анализ на алтернативите и оценка на тяхната релевантност спрямо конкретните цели и контекст, в който се извършва оценката.

Налице са изследвания, които съдържат данни и оценки за различни елементи на достьпа до здравни услуги в България (Balabanova and McKee, 2002; Atanasova, Rohova and Dimova, 2016; OECD/European Observatory on Health Systems and Policies, 2017; Dimova et al., 2018) или в отделни региони на страната (Rohova, Dimova and Atanasova, 2017), но липсват такива, които да се фокусират върху цялостната му оценка на национално ниво.

Целта на настоящото изследване е да се извърши оценка на достьпа до здравни услуги в България. Оценката обхваща най-често изследваните и релевантни за България елементи на 
достъпа до здравни услуги и е базирана на група от показатели за всеки от тях, намиращи най-голямо приложение в международен план.

\section{1. Материал и методи}

За извършването на настоящата оценка на достъпа до здравни услуги в България е приложена методика, състояща се от пет последователни етапа:

1) Анализирани са 26 литературни източници, съдържащи показатели за измерване на достъпа до здравни услуги на национално ниво, сред които 17 национални рамки за оценка на функционирането на здравната система и 9 доклада на международни организации и авторски колективи, свързани с оценка на функционирането на здравната система. В анализираните източници са идентифицирани общо 302 показатели, които са използвани или са били определени като подходящи за измерване на достъпа до здравни услуги.

2) Показателите са групирани по елементи на достьпа, които се изследват чрез тях. На тази основа са определени трите елемента с най-много показатели за тяхното измерване.

3) Идентифицирани са най-често използваните показатели за оценка на всеки от тези три елементи на достьпа.

4) На база проучване на статистически бази данни, статистически справочници, бюлетини, доклади и научни публикации са събрани наличните официални данни за идентифицираните показатели. При липса на официални данни за България за даден показател и в случай, че е подходящо, той е заменен със сходен, за който такива данни са налични.

5) Данните за показателите са анализирани в национален и международен контекст и са използвани за оценка на съответния елемент на достьпа.

\section{2. Резултати и дискусия}

Както е разгледано по-горе, налице са различни виждания за това кои елементи на достъпа да бъдат изследвани при оценката му на национално ниво. В проучените национални рамки, доклади и научни публикации също липсва консенсус по този въпрос. Не във всички изследвания се наблюдава групиране на показателите за достьп спрямо различните му елементи. Най-често използвана е комбинацията между физическата наличност на материални и човешки ресурси за оказване на здравни услуги (physical availability), финансовата достъпност (financial affordability) и навременност при оказване на услугите (timeliness) (den Berg et al., 2015; Vrijens et al., 2016; Devos et al., 2019). Извършеното групиране на идентифицираните 302 показатели разкрива, че най-голям брой от тях спадат именно към тези три елемента - физическа наличност - 93, финансова достъпност - 83 и навременност - 69. Останалите 57 се отнасят към други елементи на достьпа. Анализът на показателите откроява и тези от тях, които намират най-широко приложение при оценката на всеки от трите най-изследвани елементи.

Показателите за настоящата оценка са подбрани на база на тяхната надеждност и наличието на данни за България. Проучени са официални източници на информация като електронните бази на Евростат и Световната здравна организация (С3O), научни публикации и доклади на международни организации. Показателите, за които не са открити данни, са заменени с такива, осигуряващи сходна информация.

Неудовлетворените нужди от различни здравни услуги са сред най-често срещаните показатели за измерване на достъпа. Такива са използвани за оценка на всеки от изследваните елементи. Данните за тях са получени на база на проучването European Union Statistics on Income and Living Conditions (EU SILC) и са представени от Евростат (Eurostat, 2018e).

Най-често срещаните показатели за измерване на физическата наличност са свързани с осигуреността с човешки и физически ресурси. Съществуват различни вариации, в които 
тези показатели се изчисляват. Примери за това са практикуващи или регистрирани медицински специалисти, техният абсолютен брой, население на един медицински специалист, брой медицински професионалисти или други ресурси отнесен към 100000 души население и др. За постигане на сравнимост в международен план в настоящата оценка са използвани данни от Световната здравна организация за осигуреността с ресурси спрямо 100000 души население (World Health Organisation Regional Office for Europe, 2018).

В таблица 1 е представена информация за осигуреността с човешки и материални ресурси в здравната ни система, наличните сравнителни данни в международен план, годината, за която данните се отнасят, и използвания източник.

Таблица 1. Показатели за осигуреност с човешки и материални ресурси на 100000 души от населението

\begin{tabular}{|c|c|c|c|c|c|}
\hline & \multicolumn{3}{|c|}{ Данни на СЗО } & \multicolumn{2}{|c|}{ Данни на Евростат } \\
\cline { 2 - 6 } Показател & България & $\begin{array}{c}\text { Сравнителни } \\
\text { данни - ЕС 28 }\end{array}$ & Година & България & Година \\
\hline $\begin{array}{c}\text { Брой практикуващи } \\
\text { лекари (на 100 000 от } \\
\text { населението) }\end{array}$ & 400 & 351 & 2014 & 425 & 2017 \\
\hline $\begin{array}{c}\text { Брой практикуващи } \\
\text { медицински сестри (на } \\
100 \text { 000 от населението) }\end{array}$ & 487 & 868 & 2014 & 438 & 2017 \\
\hline $\begin{array}{c}\text { Брой } \\
\text { общопрактикуващи } \\
\text { лекари (на 100 000 от } \\
\text { населението) }\end{array}$ & 63 & 80 & 2014 & 64 & 2017 \\
\hline $\begin{array}{c}\text { Брой практикуващи } \\
\text { лекари по дентална }\end{array}$ & 98 & 68 & 2014 & 118 & 2017 \\
$\begin{array}{c}\text { медицина (на 100 000 от } \\
\text { населението) }\end{array}$ & & 521 & 2014 & 745 & 2017 \\
\hline $\begin{array}{c}\text { Брой на болничните } \\
\text { легла (на 100 000 от } \\
\text { населението) }\end{array}$ & 715 & & & \\
\hline
\end{tabular}

Анализ на данните от таблица 1 показва осигуреност над средната за ЕС с общ брой практикуващи лекари, практикуващи лекари по дентална медицина и болнични легла на 100000 души население. В същото време се отчитат 21\% по-малко общопрактикуващи лекари и 44\% по-малко медицински сестри на 100000 души население спрямо средните за EC нива. Данни за България на Eurostat за 2017 г. не сочат значителни изменения в стойностите на повечето показатели спрямо отчетените от СЗО за 2014 г. България заема едни от последните места по брой практикуващи медицински сестри и по брой общопрактикуващи лекари на 100000 души население, в сравнение с останалите страни от EC (Eurostat, 2018c, 2018b, 2018d, 2018a). Липсата на достатъчно общопрактикуващи лекари, особено в отдалечените райони и устойчивият във времето недостиг на медицински сестри (Dimova et al., 2018) могат да бъдат сериозна бариера пред наличността на здравните услуги. В списъка от показатели, служещи за оценка на физическата наличност, като елемент на достьпа до здравни услуги попадат и два показателя за равнището на неудовлетворените нужди поради голямо разстояние. Неудовлетворени нужди от медицински преглед/лечение поради голямо разстояние, са декларирали $0,3 \%$ от интервюираните българи, при средно 
0,1\% за ЕС. Неудовлетворени нужди от дентални услуги поради голямо разстояние са отчетени при $0,1 \%$ от българите. Средната стойност на този показател в ЕС е представена като несъществена - 0. Въпреки че отдалечеността не се явява значителен фактор за възникване на неудовлетворени нужди от лечение или дентални услуги, равнищата на тези показатели за България превишават средните за ЕС.

В таблица 2 са представени най-често използваните показатели за оценка на финансовата достьпност, данни за сравнение в международен план, годината, за която се отнасят, и източникът на информация.

Таблица 2. Показатели за оценка на финансовата достьпност

\begin{tabular}{|c|c|c|c|c|}
\hline Показател & България & $\begin{array}{l}\text { Сравнителни } \\
\text { данни - ЕС } 28\end{array}$ & Година & Източник \\
\hline $\begin{array}{c}\text { Директни плащания на } \\
\text { домакинствата за } \\
\text { здравеопазване (дял от } \\
\text { потреблението на } \\
\text { домакинството) } \\
\end{array}$ & $6.30 \%$ & $4.00 \%$ & 2017 & Eurostat \\
\hline $\begin{array}{c}\text { Директни плащания на } \\
\text { домакинствата за } \\
\text { здравеопазване (дял от } \\
\text { всички разходи за } \\
\text { здравеопазване в } \\
\text { страната) } \\
\end{array}$ & $47.96 \%$ & $21.97 \%$ & 2016 & Eurostat \\
\hline $\begin{array}{c}\text { Дял на } \\
\text { здравноосигуреното } \\
\text { население } \\
\end{array}$ & $88.20 \%$ & $97.30 \%$ & 2013 & OECD \\
\hline $\begin{array}{c}\text { Дял на домакинствата с } \\
\text { "катастрофални" } \\
\text { здравни разходи (над } \\
40 \% \text { от доходите на } \\
\text { домакинството след } \\
\text { приспадане на } \\
\text { разходите за храна) } \\
\end{array}$ & $2.00 \%$ & - & 2000 & Xu et al., 2003 \\
\hline $\begin{array}{c}\text { Неизпълнени рецепти } \\
\text { поради финансови } \\
\text { причини } \\
\end{array}$ & $9.60 \%$ & $4.60 \%$ & 2014 & Eurostat \\
\hline $\begin{array}{c}\text { Неудовлетворени нужди } \\
\text { от медицински } \\
\text { преглед/лечение поради } \\
\text { финансови причини } \\
\end{array}$ & $1.60 \%$ & $1.00 \%$ & 2017 & Eurostat \\
\hline $\begin{array}{c}\text { Неудовлетворени нужди } \\
\text { от дентални услуги } \\
\text { поради финансови } \\
\text { причини }\end{array}$ & $2.70 \%$ & $2.60 \%$ & 2017 & Eurostat \\
\hline
\end{tabular}

Данните за България са по-неблагоприятни от сравнителните данни за почти всички показатели, отнасящи се до финансовата достъпност като елемент на достъпа до здравни 
услуги. Следва да се отчете, че България е на предпоследно място сред страните от ЕС по дял на здравноосигуреното население (OECD/European Union, 2018). Тревожна е и констатацията, че делът на неизпълнените рецепти в България поради финансови причини е над два пъти по-голям от средния за ЕС. В страната се наблюдава най-високият за ЕС дял на директните плащания на домакинствата от общите разходи за здравеопазване - близо $48 \%$ (Eurostat, 2019). Това е над два пъти повече от средното ниво за ЕС и представлява съществена бариера, ограничаваща достьпа до здравни услуги, особено за по-уязвимите групи от населението (Rohova, 2017). Посочените в таблица 1 данни за дела на домакинствата с „катастрофални“ здравни разходи в България, се отнасят за 2000 г., като отчетеният процент е определен като висок за Европа (Xu et al., 2003). Изследване на С3О посочва нива за България над 2\% (World Health Organisation, 2016). Сравнителни данни за страните от ЕС-18, отнасящи се за години в периода 2011-2016, сочат средно ниво от 5,1\% (OECD Health Division, 2018). С цел по-добра оценка на показателя в динамика и удачното му сравнение в международен план, е препорьчително да се измерят по-актуални данни за неговото равнище в страната. В допълнение, нивата на директните плащания на домакинствата за здравеопазване у нас са далеч над препоръчания в доклад на СЗО праг от $15 \%$, характеризиращ здравните системи, осигуряващи солидна финансова защита на населението (Thomson, Cylus and Evetovits, 2019). Тези фактори обуславят и по-високите, в сравнение със средните за ЕС, нива на неудовлетворени нужди от медицински и дентални услуги поради финансови причини. В заключение следва да се отбележи необходимостта от адекватни мерки, които да са насочени към ограничаване на финансовите бариери пред достъпа до здравни услуги в България.

Данните за оценка на навременността като елемент на достьпа до здравни услуги са представени в таблица 3.

Таблица 3. Показатели за оценка на навременността на достьпа до здравни услуги

\begin{tabular}{|c|c|c|c|c|}
\hline Показател & България & $\begin{array}{c}\text { Сравнителни } \\
\text { данни }\end{array}$ & Година & Източник \\
\hline $\begin{array}{c}\text { Възможност за планова } \\
\text { операция в рамките на } \\
90 \text { дни* }\end{array}$ & 1.538 & 2.328 & 2018 & $\begin{array}{c}\text { Euro Health } \\
\text { Consumer Index }\end{array}$ \\
\hline $\begin{array}{c}\text { Възможност за } \\
\text { компютърна томография } \\
\text { в рамките на 7 дни* }\end{array}$ & 1.727 & 2.279 & 2018 & $\begin{array}{c}\text { Euro Health } \\
\text { Consumer Index }\end{array}$ \\
\hline $\begin{array}{c}\text { Възможност за } \\
\text { започване на } \\
\text { химиотерапия/лъчетера } \\
\text { пия в рамките на 21 } \\
\text { дни* }\end{array}$ & 1.333 & 1.754 & 2018 & $\begin{array}{c}\text { Euro Health } \\
\text { Consumer Index }\end{array}$ \\
\hline $\begin{array}{c}\text { Достъп до } \\
\text { общопрактикуващ лекар } \\
\text { в рамките на същия ден* }\end{array}$ & 1.615 & 1.984 & 2018 & $\begin{array}{c}\text { Euro Health } \\
\text { Consumer Index }\end{array}$ \\
\hline $\begin{array}{c}\text { Дял на населението, } \\
\text { което не може да бъде } \\
\text { транспортирано до } \\
\begin{array}{c}\text { лечебно заведение от } \\
\text { спешен екип в рамките } \\
\text { на 30 минути }\end{array}\end{array}$ & $0.03 \%$ & - & 2014 & $\begin{array}{c}\text { Министерство на } \\
\text { Здравеопазването }\end{array}$ \\
\hline
\end{tabular}




\begin{tabular}{|c|c|c|c|c|}
\hline $\begin{array}{c}\text { Неудовлетворени нужди } \\
\text { от медицински } \\
\text { преглед/лечение поради } \\
\text { прекалено дълго време } \\
\text { за чакане** }\end{array}$ & $0.1 \%$ & $0.7 \%$ & 2017 & Eurostat \\
\hline
\end{tabular}

Забележка:

*Съобразно методиката на проучването, на което се базират, данните за тези показатели изразяват средноаритметичната оценка на възможността за осигуряване на достъп до здравната услуга в определения времеви интервал. Величината 1 означава, че тази възможност съществува при над 90\% от пациентите, 2 - възможността съществува за над 50\% от паџиентите (обикновено е налище), а 3 възможността не съществува при повече от 50\% от пациентите (обикновено не е на лице). Сравнителните данни се отнасят за изследваните в Euro Health Consumer Index 35 страни (Björnberg and Phang, 2018).

** Данните за показателя са с източник Евростат (Eurostat, 2018e). Сравнителните данни се отнасят за страните от ЕC (EC 28)

Стойностите на показателите за навременност на достьпа до здравни услуги в България са по-благоприятни спрямо средните нива, измерени при определянето на Euro Health Consumer Index. Респондентите, участвали в това проучване, са посочили, че вероятността българските пациенти да получат достьп до здравните услуги в определените целеви времеви интервали е налице в по-голямата част от случаите. Заслужава да се отбележи устойчивостта на тези резултати във времето. База за това заключение са задоволителните данни по тези показатели за България и в изданието на изследването от 2015 г. (Björnberg, 2015).

Най-неблагоприятна спрямо средната за ЕC е оценката на показателят за достьп до общопрактикуващ лекар в рамките на същия ден. Това, в комбинация с отчетената сравнително ниска наситеност с общопрактикуващи лекари и регионалните диспропорции в разпределението им, може да се разглежда като индикация за наличието на бариери пред навременния достъп до първична медицинска помощ.

Друг показател, който определя възможностите за навременен достъп до здравни услуги, е делът на населението, за което не е налице възможност за транспортиране до лечебно заведение от екип на спешна медицинска помощ в рамките на определено време. Съществуват различни вариации на този показател, като разликата е предимно в избраното пределно време - 20, 30, 45 минути (Knowles, Leighton and Stinson, 1997; den Berg et al., 2015; Devos et al., 2019). Данни за този показател за България са представени в Концепция за развитие на спешната медицинска помощ. Не са открити съпоставими данни за средните нива за ЕС. Делът на населението, за което не е налице възможност за транспортиране до лечебно заведение от екип на спешна медицинска в рамките на 30 минути $-0,03 \%(\mathrm{MoH}$, 2014), може да бъде определен като неголям.

В подкрепа на констатацията за наличие на навременен достьп до здравни услуги в България е и ниският дял на респондентите, които посочват времето за чакане като причина за неудовлетворени здравни нужди - 0,1\% за България при $0,7 \%$ средно за ЕC (Eurostat, 2018e).

\section{Заключение}

Настоящата оценка на достъпа до здравни услуги в България е основана на синтезиран набор от някои от най-често използваните показатели, за които са налице официални и публично достъпни данни. Анализът на данните дава възможност да се заключи, че не са налице съществени бариери, които да ограничават навременния достъп до здравни услуги. Като основни бариери пред достъпа до здравни услуги в България действат високите нива на частните разходи за здравеопазване. Внимание заслужават и показателите отчитащи недостатъчна осигуреност с медицински сестри и общопрактикуващи лекари.

Данните за използваните показатели са средни за страната и по тази причина не могат да служат за идентифициране на бариери, водещи до несправедливи неравенства, породени 
от регионални, социално-икономически и други фактори. В тази връзка, предмет на бъдещи изследвания би могла да бъде оценката на достьпа до здравни услуги на определени групи от населението и в отделни региони на страната.

Интервенциите, оказващи влияние върху равнищата на достьпност до здравните услуги, влияят и върху други аспекти на здравната система. Това предполага координиране на мерките и извършването им в контекста на цялостното ѝ функциониране.

\section{References}

1. Aday, L. A. and Andersen, R. (1974) 'A framework for the study of access to medical care.', Health services research, 9(3), pp. 208-220.

2. Atanasova, E., Rohova, M. and Dimova, A. (2016) 'Unmet needs for health care services in Bulgaria', Journal of IMAB - Annual Proceeding (Scientific Papers), 22(3), pp. 1324-1327. doi: 10.5272/jimab.2016223.1324.

3. Balabanova, D. and McKee, M. (2002) 'Access to health care in a system transition: The case of Bulgaria', International Journal of Health Planning and Management, 17(4), pp. 377-395. doi: 10.1002/hpm.687.

4. den Berg, M. J. et al. (2015) Dutch health care performance report 2014. Bilthoven: National Institute for Public Health and the Environment (RIVM).

5. Björnberg, A. (2015) Euro Health Consumer Index 2015. Stockholm: Health Consumer Powerhouse.

6. Björnberg, A. and Phang, A. Y. (2018) Euro Health Consumer Index 2018. Stockholm: Health Consumer Powerhouse.

7. Braithwaite, J. et al. (2017) 'Health system frameworks and performance indicators in eight countries: A comparative international analysis', SAGE Open Medicine, 5. doi: $10.1177 / 2050312116686516$.

8. Clark, D. W. (1983) 'Dimensions of the concept of access to health care', Bulletin of the New York Academy of Medicine: Journal of Urban Health, 59(1), pp. 5-8.

9. Commission of the European Communities (2009) Communication from the commission to the European parliament, the Council, the European economic and social committee and the Committee of the regions. Solidarity in health: reducing health inequalities in the EU, COM 567 final. Available at: https://eur-lex.europa.eu/legalcontent/EN/TXT/PDF/?uri=CELEX:52009DC0567\&from=EN. .

10. Devos, C. et al. (2019) Performance of the Belgian health system - report 2019. KCE Report. Brussels: Belgian Health Care Knowledge Centre (KCE). doi: D/2019/10.273/34.

11. Dimova, A. et al. (2018) Bulgaria: Health system review, Health systems in transition. World Health Organization (acting as the host organization for, and secretariat of, the European Observatory on Health Systems and Policies). Available at: http://www.euro.who.int/_data/assets/pdf_file/0005/383054/HiT-Bulgaria-2018web.pdf?ua $=1$.

12. Eurostat (2018a) Healthcare personnel statistics - dentists, pharmacists and physiotherapists, Eurostat Statistics Explained. Available at: https://ec.europa.eu/eurostat/statisticsexplained/index.php?title=Healthcare_personnel_statistics__dentists,_pharmacists_and_physiotherapists (Accessed: 1 October 2019).

13. Eurostat (2018b) Healthcare personnel statistics - nursing and caring professionals, Eurostat Statistics Explained. Available at: https://ec.europa.eu/eurostat/statisticsexplained/index.php?title=Healthcare_personnel_statistics_-_nursing_and_caring_professionals (Accessed: 1 October 2019).

14. Eurostat (2018c) Healthcare personnel statistics - physicians, Eurostat Statistics Explained. Available at: https://ec.europa.eu/eurostat/statisticsexplained/index.php?title=Healthcare_personnel_statistics_-_physicians\#Healthcare_personnel (Accessed: 1 October 2019). 
15. Eurostat (2018d) Healthcare resource statistics - beds, Eurostat Statistics Explained. Available at: https://ec.europa.eu/eurostat/statisticsexplained/index.php?title=Healthcare_resource_statistics_-_beds (Accessed: 1 October 2019).

16. Eurostat (2018e) Unmet health care needs statistics, Eurostat Statistics Explained. Available at: https://ec.europa.eu/eurostat/statistics-

explained/index.php/Unmet_health_care_needs_statistics\#Unmet_needs_for_medical_examinat ion_or_treatment (Accessed: 1 October 2019).

17. Eurostat (2019) Out-of-pocket expenditure on healthcare, Eurostat Data Browser. Available at: https://ec.europa.eu/eurostat/databrowser/view/tepsr_sp310/default/table?lang=en (Accessed: 1 October 2019).

18. Evans, D. B., Hsu, J. and Boerma, T. (2013) 'Universal health coverage and universal access', Bulletin of the World Health Organization. doi: 10.2471/BLT.13.125450.

19. Knowles, J., Leighton, C. and Stinson, W. (1997) Measuring results of health sector reform for system performance: a handbook of indicators. Available at: http://citeseerx.ist.psu.edu/viewdoc/download?doi=10.1.1.202.795\&rep=rep1\&type=pdf.

20. Ministry of Health (2014) Concept for the development of emergency medical care in the Republic of Bulgaria. Sofia. Available at: https://www.mh.government.bg/media/filer_public/2015/04/15/kontseptsiya-razvitie-naspeshnata-meditsinska-pomosht_26-11-2014.pdf, (in Bulgarian).

21. Murray, C. and Frenk, J. (2000) 'A framework for assessing performance of health system', Bulletin of the World Health Organization, 78(6), pp. 717-31. Available at: https://www.who.int/bulletin/archives/78(6)717.pdf.

22. OECD/European Observatory on Health Systems and Policies (2017) Bulgaria: Country health profile 2017, State of health in the EU. Paris/Brussels: OECD Publishing//European Observatory on Health Systems and Policies. doi: http://dx.doi.org/10.1787/9789264283305-en.

23. OECD/European Union (2018) Health at a glance: Europe 2018: State of health in the EU Cycle. Paris: OECD Publishing. doi: 10.1787/health_glance_eur-2018-en.

24. Penchansky, R. and Thomas, J. W. (1981) 'The concept of access: Definition and relationship to consumer satisfaction', Medical Care, 19(2), pp. 127-40. doi: 10.1097/00005650-19810200000001.

25. Rohova, M. (2017) 'Private health expenditures and inequities in access to health services in Bulgaria', Varna Medical Forum, 6(1), pp. 177-183. Available at: http://journals.muvarna.bg/index.php/vmf/article/view/1873, (in Bulgarian).

26. Rohova, M., Dimova, A. and Atanasova, E. (2017) 'Access to healthcare services in The Northeast region of Bulgaria', in First National Conference '39. Public Health - A Global Priority in Science and Practice'. Varna, pp. 66-73, (in Bulgarian).

27. Saurman, E. (2016) 'Improving access: Modifying penchansky and thomas's theory of access', Journal of Health Services Research and Policy, 21(1), pp. 36-39. doi: 10.1177/1355819615600001.

28. Thomson, S., Cylus, J. and Evetovits, T. (2019) Can people afford to pay for health care? New evidence on financial protection in Europe. Copenhagen. Available at: http://apps.who.int/bookorders.

29. Vrijens, F. et al. (2016) Performance of the Belgian health system - report 2015 - Health Services Research (HSR). KCE Report. Brussels: Belgian Health Care Knowledge Centre (KCE). doi: D/2016/10.273/03.

30. World Health Organisation (2016) World health statistics 2016. Monitoring health for the SDGs. Geneva: World Health Organization. doi: 10.1017/CBO9781107415324.004.

31. World Health Organisation Regional Office for Europe (2018) European database on human and technical resources for health, European Health for All database. Available at: https://gateway.euro.who.int/en/datasets/european-health-for-all-database/ (Accessed: 4 October 2019). 